\title{
Rescate del cultivo de Goldenberry (Physalis peruviana) en los Andes del norte de Chile
}

\author{
Rescue of the cultivation of Goldenberry (Physalis peruviana) \\ in the Andes of northern Chile \\ Luke Crosley' ${ }^{1}$, José Mario Henríquez², Felipe Parra², Patricia Pacheco², \\ Hugo Escobar ${ }^{2}$, Claudio Parra2*
}

\section{RESUMEN}

La Physalis peruviana, es un cultivo andino con una amplia distribución en América del Sur, encontrándose también en casi todos los sectores altos de los trópicos y en varias partes de los subtrópicos. En Chile, sólo se tiene registro del cultivo en el sur del país, no encontrándose ningún registro para esta especie en los Andes del extremo norte del país. Este estudio evaluó la adaptación del cultivo Physalis peruviana a las condiciones edafológicas actuales de las localidades andinas del norte de Chile. La caracterización de aguas y suelos de las zonas estudiadas cumplen con los parámetros de cultivo para esta especie. Además, se estableció un manejo agronómico según el piso ecológico, con el fin de rescatar un cultivo andino olvidado por décadas en este sector. Socoroma.

Palabras claves: Condiciones edafológicas, Goldenberry, Physalis peruviana, Parámetros físico-químicos, Saxamar,

\begin{abstract}
The Physalis peruviana, is an Andean crop with a wide distribution in South America, is also found in almost all the high sectors of the tropics and several parts of the subtropics worldwide. In Chile, there is only one record of the crop in the south of the country, and there is no evidence for this species in the northern Andes sector of the country. This study evaluated the adaptation of the P. peruviana crop to the current edaphological conditions of the Andean localities of northern Chile, in addition to the quality of the fruit. The characterization of waters and soils of the studied areas comply with the cultivation parameters for this species. Besides, agronomic management was established according to the ecological floor, to rescue an Andean crop forgotten for decades in this sector.
\end{abstract}

Keywords: Edaphological conditions, Goldenberry, Physalis peruviana, Physico-chemical parameters, Saxamar, Socoroma.

\section{Introducción}

El cultivo de Physalis peruviana está asociado a las zonas altas de Sudamérica, situando su origen en los andes peruanos (Legge, 1974). Hoy se encuentra en casi todos los altiplanos de los trópicos y en varias partes de los subtrópicos, incluyendo Malasia, China, África y el Caribe (Fisher et al., 2014). Esta planta crece de forma silvestre entre 1500 y 3000 metros sobre el nivel del mar y es semi arbustiva, anual o perenne dependiendo del clima donde es cultivado. En condiciones ideales de cultivo necesita alta luminosidad, temperaturas promedio entre 13 y 18 grados centígrados, precipitación anual de entre 1000 y 2000 milímetros y humedad relativa de 70 a $80 \%$; preferiblemente en suelos con una estructura granular y una textura areno arcillosa, que contengan altos contenidos de materia orgánica y un $\mathrm{pH}$ entre 5,5 y 6,8 (Gaitan et al., 2018). Produce un fruto esférico, comestible, con gran cantidad de semillas, envuelto en un cáliz de 5 sépalos que le otorga gran protección

\footnotetext{
1 Instituto Nacional de Desarrollo Agropecuario; INDAP-Tarapacá, Iquique, Chile.

2 Facultad de Ciencias Agronómicas, Universidad de Tarapacá. Arica, Chile.

* Autor por correspondencia: cparra@uta.cl.
} 
natural. Numerosas publicaciones confieren a esta especie propiedades beneficiosas para el ser humano, tanto medicinales como nutricionales (Fisher et al., 2012; Hassanien, 2011). Siendo una buena fuente de provitamina A, vitamina $\mathrm{C}$, complejo de vitamina $\mathrm{B}$, compuestos fenólicos y varios minerales de importancia nutricional (Pereda et al., 2019). Es por esto que el cultivo de $P$. peruviana es una alternativa de producción para la economía agraria de muchos países, debido a que presenta buenas perspectivas e interés en los mercados internacionales, lo cual se deriva de las características nutricionales que posee el fruto (Gastelum, 2017). El principal productor es Colombia, con cerca del $90 \%$ del total mundial. Se cultiva en menor escala en Sudáfrica, Ecuador, Perú, Zimbawe y México y hay experiencias menores en España, Francia, el sur de Italia, Australia, India, Reino Unido y Kenia. En Chile, hasta el año 2010, se contaba con alrededor de 5,5 ha cultivadas en las regiones centrales del país (Cruzat y Honorato, 2010), no habiendo registros de los Andes del norte de Chile, evidenciando una pérdida de biodiversidad agrícola. En este contexto, este trabajo tiene como objetivo evaluar el potencial de adaptación del cultivo $P$. peruviana a las condiciones edafológicas actuales de las localidades andinas del norte de Chile en relación al crecimiento, rendimiento y calidad del fruto con el fin de promover su rescate y comercialización.

\section{Caracterización físico-químico de agua y suelo}

En este trabajo se seleccionaron las localidades de Saxamar (latitud S18³3.208'; longitud O069 29.538') y Socoroma (latitud S18 $15.464^{\prime}$;

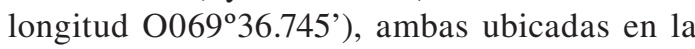
comuna de Putre, Región de Arica y Parinacota a 3308 y 2945 msnm, respectivamente. Según los parámetros establecidos por Almanza y Fischer (Almanza y Fisher, 2011), el agua de riego y el suelo analizados fueron adecuados para el cultivo de esta especie (Tabla 1 y 2). El suelo tenía una textura franco arenosa con un $\mathrm{pH}$ de 7.10 para Saxamar (SXM) y 7.42 para Socoroma (SCM). El $\mathrm{pH}$ ligeramente alcalino de los suelos no fue un impedimento para el cultivo de $P$. peruviana en ambas localidades. Además, el contenido de materia orgánica encontrado fue superior al 3\% en el caso de SCM y al 2,45\% para SXM, alcanzando los niveles mínimos a los recomendados (Gaitan et al., 2018).

\section{Manejo Agronómico}

El suelo fue trabajado con un subsolador para romper la compactación superficial para facilitar la infiltración del agua de riego hacia la zona de las raíces, mejorar el crecimiento radicular, la absorción de agua y nutrientes, la aireación del suelo, aumenta el intercambio gaseoso a nivel de raíces, así como

Tabla 1. Características fisicoquímicas del agua de riego utilizada en las localidades seleccionadas.

\begin{tabular}{|c|c|c|c|c|c|c|c|c|c|c|c|c|}
\hline \multirow{2}{*}{ Localidad } & \multirow{2}{*}{$\begin{array}{c}\mathrm{CE} \\
(\mathrm{dS} / \mathrm{m})\end{array}$} & \multirow{2}{*}{$\mathrm{pH}$} & $\mathrm{Ca}^{2+}$ & $\mathrm{Mg}^{2+}$ & $\mathrm{K}^{+}$ & $\mathrm{Na}^{+}$ & $\mathrm{Cl}^{-}$ & $\mathrm{SO}_{4}^{2-}$ & $\mathrm{NO}_{3}^{-}$ & $\mathrm{HCO}_{3}^{-}$ & $\mathrm{PO}_{4}{ }^{2-}$ & B \\
\hline & & & \multicolumn{10}{|c|}{$\mathrm{mg} / \mathrm{L}$} \\
\hline Saxamar & $1,14^{\mathrm{a}}$ & $7,37^{a}$ & $146,93^{a}$ & $27,09^{a}$ & $5,71^{\mathrm{a}}$ & $37,68^{a}$ & $34,82^{a}$ & $442,01^{\mathrm{a}}$ & $14,84^{a}$ & $46,40^{\mathrm{a}}$ & $14,52^{\mathrm{a}}$ & $0,82^{\mathrm{a}}$ \\
\hline Socoroma & $0,83^{\mathrm{b}}$ & $7,51^{\mathrm{a}}$ & $86,81^{b}$ & $12,94^{b}$ & $2,59^{\mathrm{b}}$ & $56,51^{\mathrm{b}}$ & $69,58^{b}$ & $148,33^{\mathrm{b}}$ & $0,70^{\mathrm{b}}$ & $150,71^{\mathrm{b}}$ & $27,03^{b}$ & $1,6^{\mathrm{b}}$ \\
\hline
\end{tabular}

* Los valores marcados con la misma letra no muestran diferencias significativas $(p<0,05)$; CE: Conductividad eléctrica.

Tabla 2. Análisis elemental total, bases intercambiables y reacción del suelo de las localidades seleccionadas.

\begin{tabular}{|c|c|c|c|c|c|c|c|c|c|c|c|c|c|c|c|c|}
\hline \multirow{2}{*}{ Localidad $^{1,2}$} & \multirow{2}{*}{$\begin{array}{c}\mathrm{CE} \\
(\mathrm{dS} / \mathrm{m})\end{array}$} & \multirow{2}{*}{$\mathrm{pH}$} & \multirow{2}{*}{$\begin{array}{c}\text { DA } \\
(\mathrm{g} / \mathrm{cc})\end{array}$} & \multirow{2}{*}{$\begin{array}{l}\text { Sat. } \\
(\%)\end{array}$} & \multirow{2}{*}{$\begin{array}{c}\mathrm{MO}^{3} \\
(\%)\end{array}$} & $\mathrm{Ca}^{2+} \mathrm{Mg}^{2+}$ & $\mathrm{K}^{+}$ & $\mathrm{Na}^{+}$ & CEC & $\mathrm{N}^{4}$ & $\mathrm{P}$ & K & $\mathrm{Cu}$ & $\mathrm{Mn}$ & $\mathrm{Zn}$ & $\mathrm{Fe}$ \\
\hline & & & & & & \multicolumn{4}{|c|}{ meq/100g } & \multicolumn{7}{|c|}{$\mathrm{mg} / \mathrm{Kg}$} \\
\hline & 0 & $7,42^{\mathrm{a}}$ & $1,34^{\mathrm{a}}$ & $j^{\mathrm{a}}$ & 2 & & & $1^{\mathrm{a}}$ & & & & & 31 , & & & \\
\hline ocoroma & $1,22^{\mathrm{b}}$ & $7,10^{\mathrm{b}}$ & $1,09^{\mathrm{b}}$ & $42,10^{\mathrm{b}}$ & $3,40^{\mathrm{b}}$ & $20,51^{\mathrm{b}}$ & $0,93^{\mathrm{b}}$ & $2,62^{b}$ & $24,10^{\mathrm{b}}$ & $6,91^{\mathrm{b}}$ & $10,14^{b}$ & $298,52^{\mathrm{b}}$ & $3,00^{\mathrm{b}}$ & $199,80^{\mathrm{b}}$ & $5,40^{\mathrm{b}}$ & $20,60^{b}$ \\
\hline
\end{tabular}

CE: Conductividad eléctrica; DA: Densidad Aparente; Sat.: Saturación del suelo; ${ }^{1}$ Muestreo entre 15-30 cm; ${ }^{2}$ Extracción en agua 1: $2.5 ;{ }^{3}$ Digestión húmeda; ${ }^{4}$ Kjeldahl.

* Los valores marcados con la misma letra no muestran diferencias significativas $(p<0,05)$. 
a la actividad microbiana sobre la materia orgánica (Zagal et al., 2002). Durante la preparación de suelo no se utilizaron fertilizantes comerciales. Se abonó con guano de pájaro (guano blanco), utilizando 100 g por golpe en cada área destinada al trasplante. Luego, el fertilizante aplicado se cubrió con suelo para evitar pérdidas por volatilidad o arrestre a causa del agua de lluvia (Zapata Pareja et al., 2002). De forma paralela, a nivel del mar, se llevó a cabo la propagación por semillas que previamente fueron tratadas con un fungicida para evitar la contaminación al momento de la germinación. La germinación se realizó con 900 semillas en 10 placas Petri durante 14 días, las que presentaron un $99 \%$ de germinación. Posteriormente, se realizó el trasplante en spleedling de 240 alveólos con sustrato base de turba y perlita. Después de 30 días post siembra, se trasplantó a contenedores plásticos de polietileno para continuar con el crecimiento y desarrollo del cultivo a nivel del mar. Cuando las plantas de $P$. peruviana alcanzaron los 15 a $20 \mathrm{~cm}$ de alto, éstas fueron distribuidas equitativamente y trasladadas a las localidades andinas. Antes del trasplante, las plantas se mantuvieron en contenedores plásticos durante cuatro días para adaptarlas a su nuevo entorno. Luego del trasplante se observa un crecimiento lento, probablemente debido a la incrementada radiación UV y, además, las plantas comienzan a adaptarse a un suelo con características físico-químicas diferentes al sustrato inicial. En este sentido, Fischer y Lüdder (2002), indica que esta especie responde con menor crecimiento de raíces y tallo cuando la temperatura del aire y del suelo se reduce por efecto de la altura geográfica. Sin embargo, en los días siguientes se evidencian nuevos brotes en el cultivo. El marco de plantación utilizado fue de $1,5 \mathrm{~m}$ entre hilera y $1 \mathrm{~m}$ sobre hilera, este marco de plantación busca poder mantener las plantas a menor distancia para poder combatir las heladas (Gaitan et al., 2018).

Generalmente, $P$. peruviana forma ramificaciones amplias y densas, pero debido a su hábito rastrero tiende a decaer y, a raíz de esto, se debe tener un sistema de poda programado y tutorado. En la primera etapa, se aplicó una poda de formación, eliminando los brotes que se desarrollan en la base del tallo principal. Esto disminuye la humedad al interior de la zona del cultivo, como también, la aparición de enfermedades, favoreciendo la bifurcación apical natural del tallo y el desarrollo de cuatro ramas generativas principales con sus respectivas ramas reproductivas laterales (Zapata Pareja et al.,
2002). Esta especie es exigente en agua debido a su crecimiento indeterminado, y también para el adecuado desarrollo vegetativo y reproductivo, principalmente en el desarrollo (Fisher et al., 2014). El riego se aplicó por goteo una vez por semana durante tres horas con gotero de $4 \mathrm{~L} / \mathrm{h}$, además, se aplicó un bioestimulante que contiene compuestos de acción citocínica, auxínica y giberélica, además de micronutrientes y oligosacáridos a base de Ascophyllum nodosum una vez a la semana cada 14 días.

Por otra parte, las bajas temperaturas afectaron la adaptación de las plantas. Se observó que el cultivo ubicado a 3300 msnm presentó los cambios más agresivos en sus hojas, afectando el $40 \%$ del cultivo. Sin embargo, las plantas afectadas presentaron nuevos brotes básales libre del daño por las heladas. Estos resultados están acorde a los informados previamente por otros autores que relacionan las bajas temperaturas con el crecimiento de esta especie (Fisher y Lüdder, 2002). Por el contrario, los cultivos a menor altura no presentaron daños por heladas. Cada treinta días, se aplicó una poda de mantenimiento o sanitaria, eliminando todas las ramas improductivas, además de los chupones básales, hojas y cálices enfermos o atacados por insectos plaga como indica Zapata Pareja et al. (2002). Esta poda mejora el ingreso de la radiación solar y la circulación de aire; sin embargo, para las zonas estudiadas, se dejó mayor área foliar para combatir las heladas presentes en Precordillera. Finalmente, el tiempo de transición para la madurez del fruto fue de 47 días, los primeros brotes florales fueron a fines del quinto mes y las primeras cuajas ha mediado del sexto, para finalmente iniciar las cosechas al finalizar el octavo mes desde la germinación.

\section{Caracterización físico-químico de fruto}

El cultivo de Saxamar (SXM) mostró un rendimiento mensual por hilera de $4,04 \mathrm{~kg}$, es decir $0,0897 \mathrm{~kg} / \mathrm{m}^{2}$. El diámetro transversal del fruto alcanzó entre 12 y $16 \mathrm{~mm}$ dependiendo del vigor de la planta en producción. En relación al peso del fruto, éstos consiguieron un peso fresco de entre $\operatorname{los} 2,0$ a 3,0 gramos por unidad. Por otra parte, el cultivo ubicado en la localidad de Socoroma (SCM) presentó un rendimiento mensual por hilera de 4,64 kg. El diámetro transversal de la fruta obtenida en $\operatorname{SCM}(17,82 \pm 0,15)$ fue mayor que la fruta cultivada SXM, y su peso varió entre 3,5 y 4,5 gramos por unidad (ver Tabla 3). 
Tabla 3. Características fisicoquímicas de los frutos de $P$. peruviana cultivados en los Andes chilenos.

\begin{tabular}{lccccccc}
\hline Localidad & Peso $(\mathrm{g})$ & $\begin{array}{c}\text { Diametro } \\
\text { Transversal } \\
(\mathrm{mm})\end{array}$ & $\begin{array}{c}\text { Diametro Polar } \\
(\mathrm{mm})\end{array}$ & $\mathrm{pH}$ & $\begin{array}{c}\text { Solublidos Totales } \\
\left({ }^{\circ} \text { Brix }\right)\end{array}$ & $\begin{array}{c}\text { Acidez Titulable } \\
(\% \mathrm{~m} / \mathrm{m} \text { ác. cítrico) }\end{array}$ & $\begin{array}{c}\text { Índice de } \\
\text { Madurez }\end{array}$ \\
\hline Saxamar & $2,46 \pm 0,52^{\mathrm{a}}$ & $14,10 \pm 0,22^{\mathrm{a}}$ & $17,30 \pm 0,13^{\mathrm{a}}$ & $3,71 \pm 0,02^{\mathrm{a}}$ & $14,2 \pm 0,04^{\mathrm{a}}$ & $2,23 \pm 0,02^{\mathrm{a}}$ & $6,37 \pm 0,02^{\mathrm{a}}$ \\
Socoroma & $3,99 \pm 0,55^{\mathrm{a}}$ & $17,82 \pm 0,15^{\mathrm{b}}$ & $18,12 \pm 0,89^{\mathrm{a}}$ & $3,55 \pm 0,09^{\mathrm{a}}$ & $14,6 \pm 0,06^{\mathrm{a}}$ & $2,46 \pm 0,21^{\mathrm{a}}$ & $6,06 \pm 0,05^{\mathrm{b}}$ \\
\hline
\end{tabular}

* Todas las muestras se realizaron por triplicado $( \pm \mathrm{SD})$

** Los valores marcados con la misma letra no muestran diferencias significativas $(p<0.05)$.

Las propiedades fisicoquímicas medidas de los frutos cultivados en SXR y SCM se muestran en la Tabla 3. Con respecto al peso promedio y el diámetro transversal y longitudinal, SCM tiene valores más altos que SXR. Esta diferencia puede atribuirse al hecho de que las frutas recolectadas en SXM sufrieron daños a las bajas temperaturas que estuvieron expuestas. SCM tenía un pH más alto y una acidez titulable (TA) más baja que SXR, verificando la relación inversa entre pH y TA en ambas frutas. Además, se encontró una ligera diferencia entre los valores de los sólidos solubles totales (SST) y el índice de madurez (IM). Los valores de SST, IM y TA para ambas muestras están en la zona media de acuerdo con los reportados por ICONTEC (ICONTEC 1999), lo que indica un estado de madurez para el consumo, diferente a los frutos del ecotipo Colombia de $P$. peruviana, lo que estaría vinculado al origen de las semillas.

\section{Conclusión}

Se rescató un cultivo andino olvidado por décadas en la Precordillera del norte de Chile, adaptándolo a las condiciones edafológicas actuales. Además, se concluye que las características climáticas de Socoroma favorecen al crecimiento del cultivo y los parámetros físico-químicos del fruto de $P$. peruviana. Estos resultados pueden incentivar a pequeños agricultores a cultivar esta planta y diversificar su economía agraria.

\section{Literatura Citada}

Almanza, P.; Fischer, G.

2012. Fisiología del cultivo de la uchuva (Physalis peruviana L.). En: Anais II Reunião Técnica da Cultura da Physalis, 3-4 Abr. 2012, UDESC. Lages, Brasil. pp. 32-52.

Cruzat, R.; Honorato, C.

2010. Resultados y lecciones en Cultivo de Golden berry (Physalis peruviana $\mathrm{L}$.) en la zona central de Chile. Fundación para la Innovación Agraria (FIA). Región Maule, Chile. 58 p.

Fischer, G.; Almanza-Merchán, P.J.; Miranda, D. 2014 Importancia y cultivo de la uchuva (Physalis peruviana L.). Revista Brasileira de Fruticultura, 36 (1): 1-15.

Fischer, G.; Ebert, G.; Lüdders, P.

2012. Production, seeds and carbohydrate contents of cape gooseberry (Physalis peruviana L.) fruits grown at two contrasting Colombian altitudes. Journal of Applied Botany and Food Quality, 81 (1): 29-35.

Fischer, G.; Lüdders, $\mathrm{P}$

2002. Efecto de la altitud sobre el crecimiento y desarrollo vegetativo de la uchuva (Physalis peruviana L.). Revista Comalfi, Bogotá, 29 (1): 1-10.

Gaitan, L.M.R.; González, C.L.C.; Villamizar, C.J.

2018. El cultivo de la uchuva (Physalis peruviana L.). Revista Científica Agroecosistemas, 6 (1): 46-53.

Gastelum, D.

2012. Demanda nutrimental y manejo agronómico de Physalis peruvianum L. Institución de enseñanza e investigación en ciencias agrícolas. Colpos. Textoco, México. 74 p.

Hassanien, M.F.R.

2011. Physalis peruviana: a rich source of bioactive phytochemicals for functional foods and pharmaceuticals. Food reviews international, 27 (3): 259-273.

\section{ICONTEC}

1999. Fresh Fruits Cape Gooseberry. Specifications. Colombian Standard. NTC 4580. Bogota, Colombia. 15 p.

Legge. A.

1974. Notes on the history, cultivation and uses of Physalis peruviana L. Journal of the Royal Horticultural Society, 99(7): 310-314.

Pereda, M.S.B.; Nazareno, M.A.; Viturro, C.I.

2019. Nutritional and Antioxidant Properties of Physalis peruviana L. Fruits from the Argentinean Northern Andean Region. Plant Foods for Human Nutrition, 74 (1): 68-75.

Zagal, E.; Rodríguez, N.; Vidal, I.; Quezada, L.

2002. Actividad microbiana en un suelo de origen volcánico bajo distinto manejo agronómico. Agricultura Técnica, 62 (2): 297-309.

Zapata Pareja, J.L.; Saldarriaga Cardona, A.; Londoño Bonilla, M.; Díaz Diez, C.A.

2002. Manejo del cultivo de la uchuva en Colombia. Corporación Colombiana de Investigación AgropecuariaCORPOICA. Rionegro, Antioquia,Colombia. 40 p. 